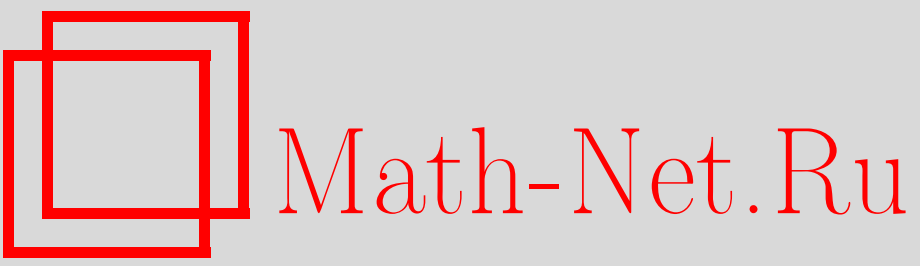

Б. Фидлер, М. И. Вишик, Количественное усреднение глобальных аттракторов гиперболических волновых уравнений с быстро осциллирующими коэффициентами, УМН, 2002, том 57, выпуск 4, 75-94

DOI: https://doi.org/10.4213/rm534

Использование Общероссийского математического портала Math-Net.Ru подразумевает, что вы прочитали и согласны с пользовательским соглашением

http://www.mathnet.ru/rus/agreement

Параметры загрузки:

IP: 54.224 .60 .19

26 апреля 2023 г., 13:03:58 


\title{
КОЛИЧЕСТВЕННОЕ УСРЕДНЕНИЕ ГЛОБАЛЬНЫХ АТТРАКТОРОВ ГИПЕРБОЛИЧЕСКИХ ВОЛНОВЫХ УРАВНЕНИЙ С БЫСТРО ОСЦИЛЛИРУЮШИМИ КОЭФФИЦИЕНТАМИ
}

\author{
М. И. ВИшИК, Б. ФИДЛЕР
}

\begin{abstract}
Оценивается скорость сходимости решений и аттракторов к соответствующим решениям и аттракторам пределного усредненного уравнения.

Библиография: 17 названий.
\end{abstract}

\section{СОДЕРЖАНИЕ}

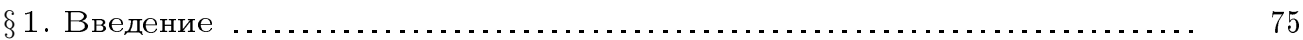

$\S 2$. Локальные и глобальные оценки роста ........................ 83

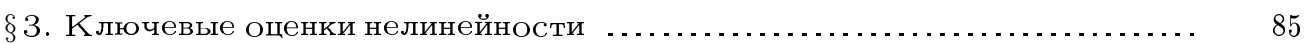

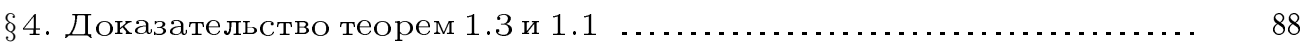

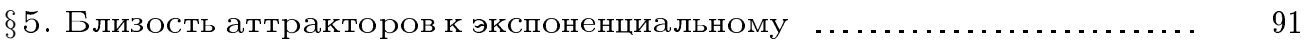

Список литературы .................................................. 94

\section{$\S$ 1. Введение}

Мы дадим количественные оценки сходимости решений гиперболических волновых уравнений с быстро осциллирующими по пространству коэффициентами к решениям предельного (усредненного) уравнения на примере следуюшего скалярного уравнения:

$$
\partial_{t}^{2} u+\gamma^{\varepsilon}(x, x / \varepsilon) \partial_{t} u=\Delta u-b(x, x / \varepsilon) f(u)+g(x, x / \varepsilon),
$$

$u \in \mathbb{R}$. Здесь $x$ принадлежит ограниченной области $\Omega \subset \mathbb{R}^{n}$ и наложены нулевые краевые условия Дирихле:

$$
u=0 \text { на } \partial \Omega .
$$

Мы предполагаем быструю сходимость диссипативного коэффициента $\gamma^{\varepsilon}$ в следующей форме:

$$
\gamma^{\varepsilon}(x, z)=\gamma^{0}(x)+\varepsilon \widetilde{\gamma}(x, z) .
$$

Наши оценки для индивидуальных траекторий будут включать случай отрицательной диссипации. Положительная диссипация $\gamma^{0}>0$ будет предполагаться лишь при количественной оценке сходимости глобальных аттракторов к глобальному аттрактору усредненного уравнения. Уравнение (1.1) возникает, например, в контексте релятивистской квантовой механики; см. [17; гл. IV. 3] и библиографию там же. Ниже 
мы наложим на $b=b(x, z)$ и $g=g(x, z)$ диофантово условие квазипериодичности по быстрой пространственной переменной $z=x / \varepsilon$. На самом деле нам необходимо лишь более слабое условие, состояшее в дивергентной представимости функций $b$ и $g$ по быстрой переменной $z$; см. (1.16)-(1.21) ниже. В случае параболических систем уравнений реакции-диффузии те же дивергентные представления (1.16)-(1.19) тоже влекут количественные оценки близости к предельному случаю, см. [7].

Хотя $\widetilde{\gamma}, b, f, g$ могут явно зависеть от $\varepsilon$, мы опускаем эту зависимость, чтобы не усложнять обозначения. Для корректной постановки задачи мы потребуем

$$
b=b(x, z) \in C^{0}\left(\bar{\Omega} \times \mathbb{R}^{n}\right), \quad 0 \leqslant b \leqslant \beta_{1}<\infty,
$$

и

$$
\begin{gathered}
\|g(\cdot, \cdot / \varepsilon)\|_{L^{2}(\Omega)} \leqslant C, \\
\left\|\gamma^{\varepsilon}(\cdot, \cdot / \varepsilon)\right\|_{L^{\infty}(\Omega)} \leqslant C
\end{gathered}
$$

равномерно при $0<\varepsilon \leqslant \varepsilon_{0}$ с некоторой константой $C$. Здесь $g(\cdot, \cdot / \varepsilon), \gamma^{\varepsilon}(\cdot, \cdot / \varepsilon)$ обозначают функции $x \mapsto g(x, x / \varepsilon), \gamma^{\varepsilon}(x, x / \varepsilon)$, которые предполагаются корректно определенными и лежашими в пространствах $L^{2}(\Omega), L^{\infty}(\Omega)$ соответственно. Неотрицательность коэффициента $b$ предполагается для того, чтобы фиксировать знак приведенного ниже условия диссипативности на нелинейность $f=f(u)$. Пусть $F$ обозначает первообразную функции $f$, т.е. $F^{\prime}(u)=f(u)$. Мы предполагаем, что

$$
F(u) \geqslant-\delta u^{2}-C_{\delta}
$$

для некоторого достаточно малого $\delta>0$. Будет достаточно взять $\delta<\frac{1}{2} \lambda_{1}(\Omega) / \beta_{1}$, где $\lambda_{1}(\Omega)$ обозначает первое собственное число оператора $-\Delta$ на $\Omega$ с нулевыми краевыми условиями Дирихле. Мы предполагаем, что нелинейность $f=f(u)$ является $C^{1}$-гладкой, и требуем полиномиальность роста производной:

$$
\left|f^{\prime}(u)\right| \leqslant C\left(1+|u|^{p-1}\right) \text { при некотором } 1 \leqslant p \leqslant \frac{n}{n-2}
$$

в случае $n=\operatorname{dim} x \geqslant 3$. Заметим, что предельньй случай $p=n /(n-2)$ соболевского вложения $H_{0}^{1} \hookrightarrow L^{n(p-1)}=L^{2 n /(n-2)}$ также допускается. Здесь и далее мы не накладьваем ограничений на степень полиномиального роста $p$ в случае размерностей $n=1,2$.

При сделанных предположениях существуют глобальные решения

$$
u=u^{\varepsilon}(t, x), \quad t \geqslant 0
$$

семейства задач Коши (1.1), (1.2) с заданными начальньми даннњми:

$$
\begin{aligned}
u & =u_{0}(x), \\
u_{t} & =v_{0}(x)
\end{aligned}
$$

при $t=0$; см. [17], [2], [3], а также $\S 2$. Задача $(1.1),(1.2)$ порождает полугруппу решений $y^{\varepsilon}=\left(u^{\varepsilon}, v^{\varepsilon}\right)=\left(u^{\varepsilon}, u_{t}^{\varepsilon}\right)=S^{\varepsilon}(t)\left(u_{0}, v_{0}\right)$ в гильбертовом пространстве

$$
E=H_{0}^{1}(\Omega) \times L^{2}(\Omega) .
$$


При некоторых дополнительных предположениях мы будем количественно изучать сходимость

$$
y^{\varepsilon} \rightarrow y^{0}
$$

этих решений в более слабых пространствах

$$
E_{-\alpha}:=H^{1-\alpha} \times H^{-\alpha},
$$

$0<\alpha \leqslant 1$, при усреднении $\varepsilon \searrow 0$ быстрой пространственной осцилляции. Напомним, что $H^{-\alpha}:=\mathscr{D}\left((-\Delta)^{-\alpha / 2}\right), \alpha \in \mathbb{R}$, обозначает семейство пространств, ассоциированных с дробными степенями оператора $-\Delta$ с нулевыми краевыми условиями Дирихле. Заметим, что $H^{0}=H=L^{2}(\Omega), H^{1}=H_{0}^{1}(\Omega), E=E_{0}, H^{2}=H^{2}(\Omega) \cap H_{0}^{1}(\Omega)$ и дуальными к этим пространствам являются $H, H^{-1}$ и т.д.

Чтобы установить сходимость, мы рассмотрим усредненное уравнение

$$
u_{t t}+\gamma^{0}(x) u_{t}=\Delta u-b^{0}(x) f(u)-g^{0}(x)
$$

в $\Omega$. Мы предполагаем, что $b^{0}, g^{0}$ сушествуют в следуюшем слабом смысле:

$$
\begin{array}{r}
\int_{\Omega} \varphi(x) \gamma^{\varepsilon}(x, x / \varepsilon) d x \underset{\varepsilon \searrow 0}{\longrightarrow} \int_{\Omega} \varphi(x) \gamma^{0}(x) d x \\
\int_{\Omega} \varphi(x) b(x, x / \varepsilon) d x \underset{\varepsilon \searrow 0}{\longrightarrow} \int_{\Omega} \varphi(x) b^{0}(x) d x \\
\int_{\Omega} \psi(x) g(x, x / \varepsilon) d x \underset{\varepsilon \searrow 0}{\longrightarrow} \int_{\Omega} \psi(x) g^{0}(x) d x
\end{array}
$$

для всех $\varphi \in L^{1}(\Omega)$ и $\psi \in L^{2}(\Omega)$. Обозначим решения усредненной задачи $(1.14)$ с теми же начальными данными (1.10) через $u^{0}(t, x)$ и положим $y^{0}=\left(u^{0}, v^{0}\right)=\left(u^{0}, u_{t}^{0}\right) \in E$.

Чтобы получить количественную оценку сверху для разности $y^{\varepsilon}(t)-y^{0}(t)$, необходимо такое же дивергентное представление для $b, g$, как и в параболическом случае, см. [7]. А именно, мы предполагаем, что существуют функции $G_{k}=G_{k}(x, z)$ такие, что

$$
g(x, z)-g^{0}(x)=\sum_{i=1}^{n} \partial_{z_{i}} G_{i}(x, z)
$$

вьполнено при $x \in \Omega \subset \mathbb{R}^{n}, z \in \mathbb{R}^{n}$. Мы предполагаем также, что неравенства

$$
\begin{gathered}
\left\|G_{i}(\cdot, \cdot / \varepsilon)\right\|_{L^{2}(\Omega)} \leqslant C, \\
\left\|\partial_{x_{i}}^{1} G_{i}(\cdot, \cdot / \varepsilon)\right\|_{L^{2 n /(n+2)}(\Omega)} \leqslant C
\end{gathered}
$$

вьполнены равномерно для $0<\varepsilon \leqslant \varepsilon_{0}$. Здесь $\partial_{x_{i}}^{1}$ обозначает частную производную по $x_{i}$ в точке $(x, z)$ от функции $(x, z) \mapsto G(x, z)$. Мы, конечно же, предполагаем, что все выражения корректно определены. Аналогично, для $b$ мы предполагаем дивергентное представление функциями $B_{i}=B_{i}(x, z)$ такое, что

$$
b(x, z)-b^{0}(x)=\sum_{i=1}^{n} \partial_{z_{i}} B_{i}(x, z)
$$


с $\varepsilon$-равномерньми оценками

$$
\begin{gathered}
\left\|B_{i}(\cdot, \cdot / \varepsilon)\right\|_{L^{\infty}(\Omega)} \leqslant C, \\
\left\|\partial_{x_{i}}^{1} B_{i}(\cdot, \cdot / \varepsilon)\right\|_{L^{n}(\Omega)} \leqslant C .
\end{gathered}
$$

Далее в $\S 3$ мы напомним достаточные условия для существования подобных дивергентных представлений для функций $b(x, z), g(x, z)$ в терминах диофантовых частот квазипериодичности по $z$. См. также [7].

Наконец, для диссипативных коэффициентов $\gamma^{\varepsilon}(x, z), \gamma^{0}(x)$ мы потребуем вьполнения условия

$$
\gamma^{\varepsilon}(x, z)-\gamma^{0}(x)=\varepsilon \widetilde{\gamma}(x, z) .
$$

Заметим, что в отличие от $b, g$, мы требуем, чтобы возмушение было порядка $\varepsilon$. Однако мы не требуем дивергентного представления для $\widetilde{\gamma}$. Взамен мы требуем бо́льшую регулярность $\gamma^{0}$ :

$$
\begin{gathered}
\left\|\gamma^{0}(\cdot)\right\|_{L^{\infty}}+\left\|\gamma^{0}(\cdot)\right\|_{W^{1, n}} \leqslant C, \\
\|\widetilde{\gamma}(\cdot, \cdot / \varepsilon)\|_{L^{\infty}}+\|\widetilde{\gamma}(\cdot, \cdot / \varepsilon)\|_{W^{1, n}} \leqslant C .
\end{gathered}
$$

В частности, эти неравенства усиливают наше предыдущее условие ограниченности $L^{\infty}$-нормы (1.6). Условия $(1.20),(1.21)$ вместе могут рассматриваться как количественная версия сходимости $\gamma^{\varepsilon} \rightarrow \gamma^{0}$ при $\varepsilon \searrow 0$.

ТеОРема 1.1. Пусть выполнены предположения (1.4)-(1.8). Предположим, что быстро осциллирующие коэффициенты $b(x, x / \varepsilon), g(x, x / \varepsilon)$ удовлетворяют условию слабой сходимости (1.15) и дивергентному представлению (1.16)-(1.19), a диссипативный коэффициент $\gamma^{\varepsilon}(x, x / \varepsilon)$ удовлетворяет условиям сходимости (1.20), (1.21). Рассмотрим решения $y^{\varepsilon}=\left(u^{\varepsilon}(t, u), u_{t}^{\varepsilon}(t, x)\right)$ семейства задач $(1.1),(1.2)$ и решение $y^{0}=\left(u^{0}(t, x), u_{t}^{0}(t, x)\right)$ усредненной задачи (1.14), (1.2) $c$ одинаковыми начальными даннылми $y_{0}=\left(u_{0}(x), v_{0}(x)\right) \in E=E_{0}=H_{0}^{1}(\Omega) \times L^{2}(\Omega)$ nри $t=0$. Зафиксируем достаточно малое $\varepsilon_{0}>0$.

Тогда для любого $0<\alpha \leqslant 1$ существуют константы $c_{0}, c_{1}, c_{2}, \varrho>0$, которие зависят от $\alpha,\left\|y_{0}\right\|_{E_{0}}$ и других параметров задач, но не зависят от $\varepsilon \in\left(0, \varepsilon_{0}\right]$, такие, что разность $y^{\varepsilon}-y^{0}$ оценивается с помощью повторной әкспоненть следующим образом:

$$
\left\|y^{\varepsilon}(t)-y^{0}(t)\right\|_{E_{-\alpha}} \leqslant \varepsilon^{\alpha} \exp \left(c_{2} \exp (\varrho t)+c_{1} t+c_{0}\right)
$$

равномерно при всех $t \geqslant 0$.

Данная оченка повторной экспонентой также справедлива для случая, когда диссипативный коэффициент $\gamma^{0}(x)$ принимает отрицательные значения.

Если же диссипативный коэффициент $\gamma^{0}(x)$ обладает строго положительной нижней гранью $\gamma^{0}(x) \geqslant \underline{\gamma}>0$, то оченка может быть улучшена до әкспоненциальной:

$$
\left\|y^{\varepsilon}(t)-y^{0}(t)\right\|_{E_{-\alpha}} \leqslant C \varepsilon^{\alpha} \exp (\varrho t)
$$

для подходящей константы $C$.

Теперь обратимся к сходимости глобальных аттракторов $\mathscr{A}^{\varepsilon} \rightarrow \mathscr{A}^{0}$, где $\mathscr{A}^{\varepsilon}$ отвечает глобальным решениям $y^{\varepsilon}=\left(u^{\varepsilon}, u_{t}^{\varepsilon}\right)$ задачи $(1.1),(1.2)$, а $\mathscr{A}^{0}$ - решениям усредненной задачи (1.14), (1.2). В [3], [17] можно найти необходимые сведения для общего 
ознакомления с глобальньми аттракторами, т.е. минимальными множествами, притягиваюшими каждое ограниченное множество. Для существования и равномерной ограниченности, а также для относительной компактности $\bigcup_{0<\varepsilon \leqslant \varepsilon_{0}} \mathscr{A}^{\varepsilon}$ в $E=E_{0}$ мы усилим наши предположения (1.7), (1.8) на нелинейность $f(u)$ и ее первообразную $F(u)$; см. также [3], [17]. Мы потребуем вьполнения неравенства

$$
f(u) u \geqslant-\delta u^{2}-C_{\delta}
$$

с некоторым $\delta$, которое, как и раньше, удовлетворяет неравенству $\delta<\frac{1}{2} \lambda_{1}(\Omega) / \beta_{1}$. Более того, мы усилим предположение на рост производной (1.8) до условия

$$
\left|f^{\prime}(u)\right| \leqslant C\left(1+|u|^{p-1}\right) \quad \text { с некоторьм } 1 \leqslant p<\frac{n}{n-2}
$$

при $n=\operatorname{dim} x \geqslant 3$. Заметим, что мы лишь отказались от предельного случая $p=$ $n /(n-2)$. В качестве упрощающего (но не существенного) условия мы потребуем вьполнения следуюшего условия Гёльдера для $f^{\prime}(u)$ :

$$
\left|f^{\prime}\left(u_{1}\right)-f^{\prime}\left(u_{2}\right)\right| \leqslant C\left(1+\left|u_{1}\right|^{\beta}+\left|u_{2}\right|^{\beta}\right)\left|u_{1}-u_{2}\right|^{\theta}
$$

с некоторыми положительными константами $C, \beta, \theta$ такими, что $\beta+\theta<2 /(n-2)$.

Мыпотребуем строгой положительности диссипативного коэффициента: $\gamma^{0}(x)>0$. Тогда для предельного уравнения (1.14) с краевыми условиями (1.2) гамильтониан

$$
\Phi\left(y^{0}\right):=\int_{\Omega}\left(\frac{1}{2}\left|\partial_{t} u^{0}\right|^{2}+\frac{1}{2}\left|\nabla u^{0}\right|^{2}+b^{0} F\left(u^{0}\right)-g^{0} u^{0}\right) d x
$$

становится функцией Ляпунова. Мы дополнительно потребуем, чтобы все стационарные решения $(U, 0) \in E_{0}$ задачи $(1.14),(1.2)$ были гиперболическими. Стационарное решение $U$ удовлетворяет уравнению

$$
0=\Delta U-b^{0} f(U)+g^{0}
$$

с нулевьми граничными условиями Дирихле. Гиперболичность означает, что линеаризованное уравнение

$$
\lambda^{2} u+\lambda \gamma^{0} u=\Delta u-b^{0} f^{\prime}(U) u
$$

обладает лишь тривиальным решением $u=0$ в $H_{0}^{1}(\Omega)$ при $\operatorname{Re} \lambda=0$. Это влечет конечность числа стационарных решений, свойство седловой точки около (каждого) стационарного решения $U_{j}$, существование конечномерного неустойчивого многообразия $W_{j}^{u} \subset E_{0}$ и конечное разложение Морса

$$
\mathscr{A}^{0}=\bigcup_{j} W_{j}^{u}
$$

глобального аттрактора $\mathscr{A}^{0}$. Как показано в [3], [6], в этом случае глобальный аттрактор $\mathscr{A}^{0}$ является экспоненциально притягиваюшим множеством:

$$
\operatorname{dist}_{E_{0}}\left(y^{0}(t), \mathscr{A}^{0}\right) \leqslant C e^{-\nu t}
$$

с некоторыми положительньми постоянными $C, \nu$, которые зависят только от $\left\|y^{0}(0)\right\| E$ (см. также $\S 5)$. 
ТЕОРема 1.2. Предположим, что справедливы предположения теоремы 1.1, a также (1.24)-(1.26). Пусть диссипативный коэффициент положителен: $\gamma^{0}>0$, и не зависит от $x$. Пусть все стационарные решения $U$ задачи (1.14), (1.2) удовлетворяют условию гиперболичности (1.29). Зафиксируем $\varepsilon_{0}>0$ и $0<\alpha \leqslant 1$.

Тогда существует константа $C>0$ такая, что неравенство

$$
\operatorname{dist}_{E_{-\alpha}}\left(\mathscr{A}^{\varepsilon}, \mathscr{A}^{0}\right):=\sup _{y \in \mathscr{A}^{\varepsilon}} \operatorname{dist}_{E_{-\alpha}}\left(y, \mathscr{A}^{0}\right) \leqslant C \varepsilon^{\alpha^{\prime}}
$$

выполнено равномерно по $0<\varepsilon \leqslant \varepsilon_{0}$. Здесь показатель $\alpha^{\prime}$ связан с показателем әкспоненциального притяжения $\nu$ к аттрактору $\mathscr{A}^{0}$ в (1.31) и показателем

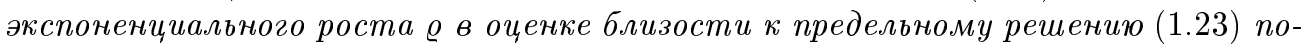
средством соотношения

$$
\alpha^{\prime}=\alpha /(1+\varrho / \nu) .
$$

Наши доказательства теорем 1.1 и 1.2 будут основываться на неравенствах гронуолловского типа, которые могут быть сформулированы в обших, абстрактных терминах сильно непрерьвных полугрупп нелинейных операторов, отвечающих полулинейным уравнениям. В качестве основы для последующих рассуждений мы сейчас опишем эти абстрактные понятия. Такой подход также может быть применен к изучению, например, гиперболических систем. Основы теории сильно непрерьвных полугруп можно найти в [13], [14], [16]. В конще настоящего введения, после формулировки абстрактной теоремы 1.3, мы кратко объясним, как наш частный пример волнового гиперболического уравнения (1.1) с краевыми условиями (1.2) укладывается в эту абстрактную схему; см. также [17]. Оценки аналитических полугрупп в пространствах с дробными показателями см. в [8].

Рассмотрим семейство банаховых пространств $E_{-\alpha}, 0 \leqslant \alpha \leqslant 1$. Предположим, что для норм $\|\cdot\|_{-\alpha}$ на $E_{-\alpha}$ справедливы интерполяционные неравенства

$$
\|y\|_{-\alpha} \leqslant C_{\alpha}\|y\|_{0}^{1-\alpha}\|y\|_{-1}^{\alpha} .
$$

Пусть $A$ порождает сильно непрерьвную полугруппу линейных операторов $\exp (A t)$, $t \geqslant 0$, в пространствах $E_{0}, E_{-1}$. При $\alpha=0,1$ мы также потребуем выполнения следующей оценки для полугрупш:

$$
\|\exp (A t)\|_{\mathscr{L}\left(E_{-\alpha}\right)} \leqslant M \exp \left(\widetilde{\varrho}_{0} t\right)
$$

при каждом $t \geqslant 0$ и подходяших постоянных $M \geqslant 1, \widetilde{\varrho}_{0} \in \mathbb{R}$. Здесь $\mathscr{L}\left(E_{-\alpha}\right)=$ $\mathscr{L}\left(E_{-\alpha}, E_{-\alpha}\right)-$ банахово пространство ограниченных линейных операторов, действующих из $E_{-\alpha}$ в $E_{-\alpha}$, с обьчной операторной нормой.

Для $0 \leqslant \varepsilon \leqslant \varepsilon_{0}$ обозначим через $y^{\varepsilon}=y^{\varepsilon}(t) \in E_{0}$ слабое решение полулинейного уравнения

$$
\frac{d}{d t} y^{\varepsilon}=A y^{\varepsilon}+\mathscr{F}_{\varepsilon}\left(y^{\varepsilon}\right)
$$

с начальньми данньми

$$
y^{\varepsilon}(0)=y_{0} \in E_{0} \text {. }
$$


Здесь и далее под слабым решением мы понимаем решение следующего интегро-операторного уравнения:

$$
y^{\varepsilon}(t)=\exp (A t) y_{0}+\int_{0}^{t} \exp (A(t-\tau)) \mathscr{F}_{\varepsilon}\left(y^{\varepsilon}(\tau)\right) d \tau \text {. }
$$

Мы будем предполагать, что слабые в этом смысле решения $y^{\varepsilon}$ существуют при всех $t \geqslant 0$, причем $y^{\varepsilon} \in C^{0}\left([0,+\infty), E_{0}\right)$, и удовлетворяют оценке не более чем экспоненциального роста

$$
\left\|y^{\varepsilon}(t)\right\|_{0} \leqslant M \exp \left(\varrho_{0} t\right)
$$

при всех $t \geqslant 0$ с подходящими константами $M \geqslant 1, \varrho_{0} \in \mathbb{R}$, которые могут зависеть от $\left\|y_{0}\right\|_{0}$, но не от $0 \leqslant \varepsilon \leqslant \varepsilon_{0}$.

Мы не будем накладьвать абстрактного условия на нелинейность

$$
\mathscr{F}_{\varepsilon}: E_{0} \rightarrow E_{0},
$$

которое гарантирует равномерность по є глобальной экспоненциальной оценки (1.39). Для большей гибкости мы наложим условие (1.39) непосредственно. В нашем частном случае гиперболического волнового уравнения (1.1) оценку (1.39) можно доказать, например, используя энергетическое неравенство; см. $\S 2$. Однако мы наложим два существенных условия на "усредненную” нелинейность $\mathscr{F}_{0}$ и разность $\mathscr{F}_{\varepsilon}-\mathscr{F}_{0}$ в более слабом пространстве $E_{-1}$ :

$$
\begin{aligned}
\left\|\mathscr{F}_{0}^{\prime}(y)\right\|_{\mathscr{L}_{\left(E_{-1}, E_{-1}\right)}} & \leqslant C\left(1+\|y\|_{0}^{p_{1}}\right), \\
\left\|\mathscr{F}_{\varepsilon}(y)-\mathscr{F}_{0}(y)\right\|_{-1} & \leqslant C\left(1+\|y\|_{0}^{p_{0}}\right) \varepsilon .
\end{aligned}
$$

Здесь $C, p_{0}, p_{1} \geqslant 1$ - подходящие константы, не зависящие ни от $\varepsilon$, ни от $y \in E_{0}$. Заметим, что условие $(1.41)$, примененное к паре $\mathscr{F}_{\varepsilon}, \mathscr{L}\left(E_{0}, E_{0}\right)$ вместо пары $\mathscr{F}_{0}$, $\mathscr{L}\left(E_{-1}, E_{-1}\right)$, влечет в силу обычной локальной липшицевой оценки локальное сушествование единственного слабого решения $y^{\varepsilon}$ в $E_{0}$. Подробности см. в $\S 2$.

ТЕОРема 1.3. Предположим, что условия (1.34), (1.35), (1.39)-(1.42) выполнены для полулинейных уравнений (1.36) и их слабьх решений $y^{\varepsilon}(t), 0 \leqslant \varepsilon \leqslant \varepsilon_{0}, c$ начальными данными $y^{\varepsilon}(0)=y_{0} \in E_{0}$.

Тогда существуют положительные константы $c_{0}, c_{1}, c_{2}$, не зависящие от $\varepsilon, \alpha$, такие, что равномерно по $0<\varepsilon \leqslant \varepsilon_{0} u 0 \leqslant \alpha \leqslant 1$ выполняется следующая повторно экспоненииальная оценка:

$$
\left\|y^{\varepsilon}(t)-y^{0}(t)\right\|_{-\alpha} \leqslant \varepsilon^{\alpha} \exp \left(c_{2} \exp (\varrho t)+c_{1} t+c_{0}\right) .
$$

Здесь $\varrho:=\varrho_{0} p\left(\varrho_{0}>0\right)$, әде $p:=\max \left(p_{0}, p_{1}\right)$, а параметры $\varrho_{0}, p_{1}$ и $p_{0}$ определяются неравенствами (1.39), (1.41) и (1.42). Зависимость $c_{j}$ от $y_{0}$ и $\alpha$ может быть явно выражена посредством констант $M, \widetilde{\varrho}_{0}, \varrho_{0}, p_{0}, p_{1}$ и $C$ следующим образом:

$$
\begin{aligned}
& c_{0}=(1-\alpha) \log (2 M)+\log C_{\alpha}, \\
& c_{1}=(1-\alpha) \varrho_{0}+\alpha\left(M C+\widetilde{\varrho}_{0}\right), \\
& c_{2}=\alpha \varrho^{-1} M^{p+1} C .
\end{aligned}
$$


В качестве замечания к теореме 1.3 мы отметим специальньй случай $\varrho=\varrho_{0}=0$, т.е. когда справедлива оценка

$$
\left\|y^{\varepsilon}(t)\right\|_{0} \leqslant M\left(y_{0}\right)
$$

для всех $t \geqslant 0$. Этот случай включает в себя консервативньй случай (без диссипации), при котором решения остаются ограниченными, а также диссипативный случай, ког да решения со временем попадают в фиксированньй большой шар.

Тогда повторно экспоненциальная оценка (1.43) может быть улучшена до экспоненциальной

$$
\left\|y^{\varepsilon}(t)-y^{0}(t)\right\|_{-\alpha} \leqslant \varepsilon^{\alpha} \exp \left(c_{1} t+c_{0}\right),
$$

где $c_{0}$ такое же, как и в $(1.44)$, а $c_{1}$ дается следующим явным выражением:

$$
c_{1}=\alpha\left(M C\left(1+M^{p}\right)+\widetilde{\varrho}_{0}\right)
$$

Доказательство следует из неравенств (4.16), (4.17) в $\S 4$.

Доказательство теоремы 1.3 приведено в $\S 4$ настоящей статьи. Опишем содержание оставшихся параграфов и схему доказательства теорем 1.1, 1.2, а также стандартную полугрупповую переформулировку гиперболического волнового уравнения (1.1) с краевыми условиями (1.2). Равномерная по $\varepsilon$ оценка экспоненциального роста (1.39) нормы $\left\|y^{\varepsilon}(t)\right\|_{0}$ в $E_{0}$ будет доказана в $\S 2$. Ключевые оценки $(1.41)$ и $(1.42)$ на $\mathscr{F}_{0}^{\prime}(y)$ и на разность $\mathscr{F}_{\varepsilon}(y)-\mathscr{F}_{0}(y)$ в пространстве $E_{-1}$ для нелинейности, соответствующей гиперболическому волновому уравнению, будут даны в $\S 3$. Вместе эти оценки позволят вьвести теорему 1.1 из теоремы 1.3. Доказательство теоремы 1.2 о количественных оценках глобальных аттракторов $\mathscr{A}^{\varepsilon}, \mathscr{A}^{0}$ будет отложено до $\S 5$.

Вернемся к стандартной полугруповой переформулировке (1.36) гиперболического волнового уравнения (1.1). Пусть $y=(u, v) \in E_{-\alpha}:=H^{1-\alpha} \times H^{-\alpha}$. Заметим, что интерполяционное неравенство (1.34) для этих пространств следует из интерполящионных неравенств в соболевских пространствах $H^{\alpha}=\mathscr{D}\left((-\Delta)^{\alpha / 2}\right)$; см. [12], [14], [16], [17]. Положим

$$
A y=A(u, v):=(v, \Delta u)
$$

с областью определения $\mathscr{D}(A)=E_{-\alpha+1}$ в $E_{-\alpha}$. Тогда $A$ порождает сильно непрерьвную полугруппу линейных операторов $\exp (A t)$ на $E_{-\alpha}$. В частности, справедлива оценка (1.35), причем с константами $M=1$ и $\widetilde{\varrho}_{0}=0$. При $\alpha=0$ это следует из того, что полугруппа $\exp (A t)$ сохраняет норму пространства $E_{0}$, которая является энергетической нормой; см. [14], [16]. Для остальных $\alpha \in \mathbb{R}$ это следует из того, что оператор $(-\Delta)^{\alpha / 2}: E_{0} \rightarrow E_{-\alpha}$ коммутирует с полугруппой $\exp (A t)$ и определяет ограниченньй линейньй изоморфизм (который можно рассматривать как изометрию) между $E_{0}$ и $E_{-\alpha}$.

Зададим нелинейность $\mathscr{F}_{\varepsilon}(y)$ в (1.36) следуюшим образом:

$$
\mathscr{F}_{\varepsilon}(u, v):=\left(0,-b^{\varepsilon} f(u)+g^{\varepsilon}-\gamma^{\varepsilon} v\right)
$$

Здесь $b^{\varepsilon}=b(x, x / \varepsilon)$ и т. д. Ясно, что соотношения $(1.48),(1.49)$ делают абстрактное полулинейное уравнение (1.36) эквивалентным полулинейному гиперболическому волновому уравнению (1.1), переписанному как система первого порядка для $(u, v)=$ $\left(u, \partial_{t} u\right)$. Поэтому сначала, в $\S 3$, мы докажем технические неравенства $(1.41),(1.42)$ 
на нелинейности $\mathscr{F}_{\varepsilon}$ и $\mathscr{F}_{0}$, а потом, в $\S 4$, мы вернемся к абстрактной теореме 1.3. Дивергентные представления (1.16)-(1.21) используются, чтобы доказать неравенства (1.41), (1.42).

Еще раз отметим, что с помощью приведенной абстрактизации мы получим повторно экспоненциальную оценку для решений гиперболического волнового уравнения, включая случаи, когда диссипативньй коэффициент $\gamma^{0}(x)$ принимает отрицательные значения. В случае постоянной положительной диссипации $\gamma^{0}>0$ мы также докажем количественную оценку для глобальных аттракторов.

Благодарности. Настоящая работа была поддержана несколькими визитами первого автора в Берлин по программе премии Александра фон Гумбольдта, которые были чрезвычайно полезны второму автору. Мы благодарны Йоргу Шмелингу за полезные обсуждения диофантовых аппроксимаций. Оба автора глубоко благодарны Регине Лёр за квалифицированньй и тщательньй набор многочисленных версий. Эта работа была также поддержана Немецким обществом исследований и Российским фондом фундаментальных исследований.

\section{§. Локальные и глобальные оценки роста}

В этом параграфе мы напомним основы теории локального существования и единственности слабых (в смысле (1.38)) решений $y^{\varepsilon}=\left(u^{\varepsilon}, \partial_{t} u^{\varepsilon}\right) \in E_{0}=H_{0}^{1} \times L^{2}$ гиперболического волнового уравнения (1.1) с нулевыми граничньми условиями Дирихле (1.2). Используемые обозначения и предположения описаны в $\S 1$. В частности, там описано сведение к абстрактному полулинейному уравнению $\frac{d}{d t} y^{\varepsilon}=A y^{\varepsilon}+\mathscr{F}_{\varepsilon}\left(y^{\varepsilon}\right)$ с начальными данньми $y^{\varepsilon}(0)=y_{0} \in E_{0}$ (см. (1.36), (1.37)). Сначала мы докажем, что для нелинейности, соответствующей уравнению (1.1), справедливо следующее условие локальной липшицевости:

$$
\left\|\mathscr{F}_{\varepsilon}^{\prime}(y)\right\|_{\mathscr{L}\left(E_{0}\right)} \leqslant C\left(1+\|y\|_{0}^{p-1}\right) .
$$

Эта оценка является ключевой для локального существования и единственности решений $y^{\varepsilon}(t)$ уравнения (1.36); см., например, [16], [14]. Потом (при предположениях $\S 1$ мы докажем глобальную экспоненциальную оценку роста (1.39) для решений уравнения (1.1) с краевьми условиями (1.2).

Напомним, что при вьводе этой оценки мы допускаем отрицательные значения диссипативного коэффициента $\gamma^{\varepsilon}(x)$. В случае положительной диссипации мы получим глобальную ограниченность решений; более того, в этом случае сушествует глобальньй аттрактор.

Для доказательства локального условия Липшица (2.1), а также для дальнейшего изложения мы отметим следуюшие простые неравенства

$$
\begin{aligned}
\|u\|_{L^{2 n /(n-2)}} & \leqslant C\|u\|_{H_{0}^{1}}, \\
\|f(u)\|_{L^{2}} & \leqslant C\left(1+\|u\|_{H_{0}^{1}}^{p}\right), \\
\left\|f^{\prime}(u)\right\|_{L^{n}} & \leqslant C\left(1+\|u\|_{H_{0}^{1}}^{p-1}\right) .
\end{aligned}
$$

Эти неравенства непосредственно следуют из оценки роста (1.8) на $f^{\prime}(u)$ и стандартного вложения Соболева $H_{0}^{1} \hookrightarrow L^{2 n /(n-2)}$. 
Докажем неравенство (2.1) в $E_{0}=H_{0}^{1} \times L^{2}$. Неравенство (2.1) вытекает из определения (1.49) нелинейности $\mathscr{F} \varepsilon$ в силу следуюших двух равномерных по $\varepsilon$ неравенств:

$$
\begin{aligned}
\left\|b^{\varepsilon} f^{\prime}(u) \widetilde{u}\right\|_{L^{2}} & \leqslant C\left(1+\|u\|_{H_{0}^{1}}^{p-1}\right)\|\widetilde{u}\|_{H_{0}^{1}}, \\
\left\|\gamma^{\varepsilon} \widetilde{v}\right\|_{L^{2}} & \leqslant C\|\widetilde{v}\|_{L^{2}} .
\end{aligned}
$$

Второе из этих неравенств является тривиальным следствием условия (1.6) равномерной по $\varepsilon$ ограниченности $L^{\infty}$-нормы коэффициента $\gamma^{\varepsilon}=\gamma^{\varepsilon}(\cdot, \cdot / \varepsilon)$. Аналогично условие $\varepsilon$-равномерной ограниченности величины $b$ константой $\beta_{1}$, наложенное в (1.4), сводит первое неравенство в (2.3) к обычному неравенству Гёльдера:

$$
\left\|f^{\prime}(u) \widetilde{u}\right\|_{L^{2}} \leqslant\left\|f^{\prime}(u)\right\|_{L^{n}}\|\widetilde{u}\|_{L^{2 n /(n-2)}} .
$$

Действительно, применяя неравенства (2.2), мы приходим к (2.3). Теперь можно воспользоваться стандартной теорией сильно непрерьвных полугруп, из которой следует локальное сушествование и единственность слабых (в смысле (1.38)) решений $y^{\varepsilon}=\left(u^{\varepsilon}, \partial_{t} u^{\varepsilon}\right) \in H_{0}^{1} \times L^{2}=E_{0}$.

Докажем глобальную экспоненциальную оценку (1.39), которая влечет сушествование и единственность глобально по времени. Доказательство будет основано на экспоненциальном неравенстве гронуолловского типа для гамильтониана

$$
\Phi\left(u, \partial_{t} u\right)=\int_{\Omega}\left(\frac{1}{2}\left|\partial_{t} u\right|^{2}+\frac{1}{2}|\nabla u|^{2}+b F(u)-g u\right) d x,
$$

введенного выше в (1.27). Дифференщируя по времени $t$, убеждаемся, что вдоль решений $\left(u, \partial_{t} u\right)$ справедливо соотношение

$$
\frac{d}{d t} \Phi\left(u, \partial_{t} u\right)=-\int_{\Omega} \gamma^{\varepsilon}\left|\partial_{t} u\right|^{2} d x
$$

В (2.6) мы пишем производную по времени от $\Phi$ лишь для удобства записи. Более точно это соотношение следует записьвать в проинтегрированной по времени форме.

Квадратическая оценка снизу (1.7) на первообразную $F$ нелинейности $f$ в свою очередь влечет

$$
\Phi\left(u, \partial_{t} u\right) \geqslant \int_{\Omega}\left(\frac{1}{2}\left|\partial_{t} u\right|^{2}+\delta^{\prime}|\nabla u|^{2}-C\right) d x
$$

при некотором $0<\delta^{\prime}<\frac{1}{2}-\beta_{1} \delta / \lambda$ и некоторой постоянной $C>0$. Мы здесь также использовали равномерную по $\varepsilon$ ограниченность (1.5) функции $g$ в $L^{2}$.

Пусть $\gamma-\varepsilon$-независимая сушественная нижняя грань диссипативного коэффициента $\gamma^{\varepsilon}(\cdot, \cdot / \varepsilon)$. Мы сначала рассмотрим случай $\gamma \leqslant 0$, которьй включает отрицательную диссипацию. Тогда соотношения $(2.6),(2 . \overline{7})$, рассмотренные вместе, влекут

$$
\frac{d}{d t}(\Phi+C) \leqslant-\underline{\gamma} \int_{\Omega}\left|\partial_{t} u\right|^{2} d x \leqslant-2 \underline{\gamma}(\Phi+C)
$$

Теперь стандартньй гронуолловский аргумент дает $\varepsilon$-равномерную экспоненщиальную оценку роста

$$
\Phi\left(y^{\varepsilon}(t)\right)+C \leqslant M^{\prime} \exp (-2 \underline{\gamma} t)
$$


Здесь $M^{\prime}=\Phi\left(y_{0}\right)+C$ зависит от $\left\|y_{0}\right\|_{E_{0}}$, но не от $\varepsilon$. Из неравенства (2.7) следует, что квадрат энергетической нормы $\left\|y^{\varepsilon}(t)\right\|_{0}^{2}$ можно оценить сверху через $\Phi+C$, из чего получаем глобальную экспоненциальную оценку роста (1.39) с $\varrho_{0}:=-\gamma$.

Следует отметить недиссипативный гамильтонов случай $\gamma^{\varepsilon} \equiv 0$, для которого $\underline{\gamma}=0$ и неравенство (1.39) преврашается в условие ограниченности константой $\bar{M}=M\left(\left\|y_{0}\right\|_{0}\right)$. В случае равномерной по $x$ и $\varepsilon$ положительности диссипативного коэффициента $\gamma^{\varepsilon}(\cdot, \cdot / \varepsilon) \geqslant \gamma>0$ гамильтонов функционал $\Phi$ становится функцией Ляпунова, которая убывает вдоль траекторий. Это дает асимптотическую ограниченность гамильтониана $\Phi$ и влечет диссипативность в $E_{0}$ в этом случае; см. также [3] и $\S 5$.

\section{§ 3. Ключевые оценки нелинейности}

В этом параграфе мы докажем ключевые неравенства (1.41), (1.42). Напомним, что эти два соотношения были предполо жены вьполненными в нашей абстрактной теореме 1.3, сформулированной для абстрактного полугруппового уравнения (1.36). Доказательство этой абстрактной теоремы будет приведено в $\S 4$. Настояший параграф посвящен доказательству того, что условия (1.41), (1.42) действительно выполнены в случае гиперболического волнового уравнения (1.1) с краевыми условиями (1.2). Напомним, что мы используем обозначения

$$
\begin{aligned}
y^{\varepsilon} & =\left(u^{\varepsilon}, v^{\varepsilon}\right), \\
E_{0} & =H_{0}^{1} \times L^{2}, \\
E_{-1} & =L^{2} \times H^{-1}, \\
\mathscr{F}_{\varepsilon}(u, v) & =\left(0,-b^{\varepsilon} f(u)+g^{\varepsilon}-\gamma^{\varepsilon} v\right) .
\end{aligned}
$$

Доказывая (1.41), (1.42), мы будем исходить из предположений теоремы 1.1 и, в частности, из дивергентных представлений (1.16)-(1.19). При таком подходе теорема 1.1 будет следовать из теоремы 1.3. В конце этого параграфа мы напомним достаточные условия дивергентных представлений (1.16)-(1.19) для случая квазипериодичных коэффициентов $b^{\varepsilon}, g^{\varepsilon}$ в терминах оценок их диофантовых частот.

Сначала мы докажем неравенство $(1.41)$, которое является $E_{-1}$-аналогом условия локальной липшицевости $(2.1),(2.3)$. Действительно, неравенство (1.41) с $p_{1}:=p-1$ следует из двух самостоятельных неравенств

$$
\begin{aligned}
\left\|b^{0} f^{\prime}(u) \widetilde{u}\right\|_{H^{-1}} & \leqslant C\left(1+\|u\|_{H_{0}^{1}}^{p-1}\right)\|\widetilde{u}\|_{L^{2}}, \\
\left\|\gamma^{0} \widetilde{v}\right\|_{H^{-1}} & \leqslant C\|\widetilde{v}\|_{H^{-1}} .
\end{aligned}
$$

Достаточно доказать эти неравенства для гладких функций $u, \widetilde{u}, \widetilde{v}$. В этом случае, в соответствии с $L^{2}$-двойственностью пространств $H^{-1}$ и $H_{0}^{1}$, норма пространства $H^{-1}$ может быть явно выражена следуюшим образом:

$$
\|\widetilde{v}\|_{H^{-1}}:=\sup _{\|\varphi\|_{H_{0}^{1}}=1} \int_{\Omega} \widetilde{v} \varphi d x .
$$

Теперь второе неравенство в (3.2) можно получить, исходя из следующей цепочки неравенств:

$$
\left|\int_{\Omega} \gamma^{0} \widetilde{v} \varphi d x\right| \leqslant\|\widetilde{v}\|_{H^{-1}}\left\|\gamma^{0} \varphi\right\|_{H_{0}^{1}} \leqslant\|\widetilde{v}\|_{H^{-1}} C\left(\left\|\gamma^{0}\right\|_{W^{1, n}}+\left\|\gamma^{0}\right\|_{L^{\infty}}\right)\|\varphi\|_{H_{0}^{1}}
$$


для гладких $\widetilde{v}, \varphi$ и для $\gamma^{0}$ из пространства Соболева $W^{1, n} \cap L^{\infty}$; см. (1.21). Аналогично, первое неравенство в (1.42) следует из неравенств Гёльдера и Соболева:

$$
\begin{aligned}
\left|\int_{\Omega} b^{0} f^{\prime}(u) \widetilde{u} \varphi d x\right| & \leqslant\left\|b^{0}\right\|_{L^{\infty}}\left\|f^{\prime}(u)\right\|_{L^{n}}\|\widetilde{u}\|_{L^{2}}\|\varphi\|_{L^{2 n /(n-2)}} \\
& \leqslant\left\|b^{0}\right\|_{L^{\infty}} C\left(1+\|u\|_{H_{0}^{1}}^{p-1}\right)\|\widetilde{u}\|_{L^{2}}\|\varphi\|_{H_{0}^{1}} \\
& \leqslant C\left(1+\|u\|_{H_{0}^{1}}^{p-1}\right)\|\widetilde{u}\|_{L^{2}}\|\varphi\|_{H_{0}^{1}}
\end{aligned}
$$

где через $C$ обозначены различные абсолютные константы; см. также (2.2). Это доказывает неравенства (3.2) и, следовательно, неравенство (1.41).

Аналогично, неравенство (1.42) с $p_{0}:=p$ сводится к следуюшим трем:

$$
\begin{aligned}
\left\|\left(b^{\varepsilon}-b^{0}\right) f(u)\right\|_{H^{-1}} & \leqslant C\left(1+\|u\|_{H_{0}^{1}}^{p}\right) \varepsilon, \\
\left\|g^{\varepsilon}-g^{0}\right\|_{H^{-1}} & \leqslant C \varepsilon \\
\left\|\left(\gamma^{\varepsilon}-\gamma^{0}\right) v\right\|_{H^{-1}} & \leqslant C\|v\|_{H^{-1}} \varepsilon
\end{aligned}
$$

для гладких $u, v$ и подходящих констант $C$. Последнее из этих трех неравенств легко следует из предположения (1.21):

$$
\begin{aligned}
\left|\int_{\Omega}\left(\gamma^{\varepsilon}-\gamma^{0}\right) v \varphi d x\right| & =\varepsilon\left|\int_{\Omega} \widetilde{\gamma} v \varphi d x\right| \leqslant \varepsilon\|v\|_{H^{-1}}\|\widetilde{\gamma} \varphi\|_{H_{0}^{1}} \\
& \leqslant C\left(\|\widetilde{\gamma}\|_{W^{1, n}}+\|\widetilde{\gamma}\|_{L^{\infty}}\right)\|v\|_{H^{-1}} \varepsilon\|\varphi\|_{H_{0}^{1}} .
\end{aligned}
$$

Оба неравенства в (3.7) обосновьваются аналогично неравенствам (3.4) с помошью предположения (1.21).

Для доказательства оставшихся двух неравенств в (3.6) мы воспользуемся дивергентными представлениями (1.16)-(1.19) для $b^{\varepsilon}-b^{0}$ и $g^{\varepsilon}-g^{0}$. Сначала рассмотрим

$$
g^{\varepsilon}-g^{0}=\left.\sum_{i=1}^{n} \partial_{z_{i}} G_{i}(x, z)\right|_{z=x / \varepsilon} .
$$

По (1.17) мы можем оценить вторую разность в (3.6) следуюшим образом:

$$
\begin{aligned}
\left|\int_{\Omega}\left(g^{\varepsilon}-g^{0}\right) \varphi d x\right| & =\left|\int_{\Omega} \sum_{i=1}^{n} \varphi \partial_{z_{i}} G_{i}(x, x / \varepsilon) d x\right| \\
& =\left|\int_{\Omega} \sum_{i=1}^{n} \varphi \cdot\left(\varepsilon \partial_{x_{i}} G_{i}(x, x / \varepsilon)-\varepsilon \partial_{x_{i}}^{1} G_{i}(x, x / \varepsilon)\right) d x\right| \\
& \leqslant \varepsilon\left(\left|\int_{\Omega} G \nabla_{x} \varphi d x\right|+\sum_{i=1}^{n}\left|\int_{\Omega} \partial_{x_{i}}^{1} G_{i} \varphi d x\right|\right) \\
& \leqslant \varepsilon\left(\|G\|_{L_{2}}\|\varphi\|_{H_{0}^{1}}+\sum_{i=1}^{n}\left\|\partial_{x_{i}}^{1} G_{i}\right\|_{L^{2 n /(n+2)}}\|\varphi\|_{L^{2 n /(n-2)}}\right) \\
& \leqslant C \varepsilon\|\varphi\|_{H_{0}^{1}} .
\end{aligned}
$$


Здесь мы снова использовали неравенства Гёльдера, соболевское вложение $H_{0}^{1} \hookrightarrow$ $L^{2 n /(n-2)}$ и обозначение $\partial_{x}^{1} G(x, z)$ для частной производной $G$ по первым $x$-компонентам, а также $\partial_{x_{i}} G$ для обозначения частной производной отображения $x \mapsto G(x, x / \varepsilon)$ по $x_{i}$. Второе неравенство в (3.6) доказано.

Для доказательства первого неравенства в (3.6) мы воспользуемся неравенствами $(2.2)$, дивергентньм представлением $(1.18),(1.19)$ разности $b^{\varepsilon}-b^{0}$ и проделаем аналогичные рассуждения:

$$
\begin{aligned}
\left|\int_{\Omega}\left(b^{\varepsilon}-b^{0}\right) f(u) \varphi d x\right| & =\left|\int_{\Omega} \sum_{i=1}^{n} \varphi \partial_{z_{i}} B_{i}(x, x / \varepsilon) f(u) d x\right| \\
& \leqslant \varepsilon\left(\left|\int_{\Omega} B \nabla_{x}(f(u) \varphi) d x\right|+\sum_{i=1}^{n}\left|\int_{\Omega} \partial_{x_{i}}^{1} B_{i} \cdot f(u) \varphi d x\right|\right) \\
& \leqslant \varepsilon\left(\| B \| _ { L ^ { \infty } } \left(\left\|f^{\prime}(u)\right\|_{L^{n}}\left\|\nabla_{x} u\right\|_{L^{2}}\|\varphi\|_{L^{2 n /(n-2)}}\right.\right. \\
& \left.\left.+\|f(u)\|_{L^{2}}\left\|\nabla_{x} \varphi\right\|_{L^{2}}\right)+\sum_{i=1}^{n}\left\|\partial_{x_{i}}^{1} B_{i}\right\|_{L^{n}}\|f(u)\|_{L^{2}}\|\varphi\|_{L^{2 n /(n-2)}}\right) \\
& \leqslant C \varepsilon\left(1+\|u\|_{H_{0}^{1}}^{p}\right)\|\varphi\|_{H_{0}^{1}} .
\end{aligned}
$$

Неравенства (3.6) и, следовательно, неравенство (1.42) доказаны.

Для удобства читателя мы завершим этот параграф несколькими замечаниями, которые связывают дивергентные представления (1.16)-(1.19) со стандартными диофантовыми условиями для случая квазипериодических коэффициентов $b^{\varepsilon}, g^{\varepsilon}$, см. также [7]. Мы укажем диофантово условие лишш для $b$, опустив аналогичное условие для $g$. Пусть справедливо соотношение

$$
b(x, z)-b^{0}(x)=\beta\left(x, \omega_{1} z_{1}, \ldots, \omega_{n} z_{n}\right)
$$

с частотными векторами $\omega_{j} \in \mathbb{R}^{\ell_{j}}$, где $\beta$ - функция, достаточно гладкая по всем своим $n+\ell:=n+\ell_{1}+\cdots+\ell_{n}$ аргументам и $2 \pi$-периодичная по всем аргументам кроме (быть может) переменной $x$.

Наложим стандартное диофантово условие КАМ-теории

$$
\left|k_{j} \omega_{j}\right| \geqslant c\left|k_{j}\right|^{-\left(\ell_{j}-1+\delta\right)}
$$

для $j=1, \ldots, n$, некоторых констант $c, \delta>0$ и всех целочисленных векторов $k_{j} \in \mathbb{Z}^{\ell_{j}} \backslash\{0\}$. Напомним, что это условие вьполняется на множестве полной лебеговой меры в пространстве частотных векторов $\left(\omega_{1}, \ldots, \omega_{n}\right) \in \mathbb{R}^{\ell}$; см., например, [4]. В работе [8], исходя из разложения Фурье

$$
\beta=\sum_{k} \beta_{k}(x) \exp \left(i \sum_{j}\left(k_{j} \omega_{j}\right) z_{j}\right),
$$

где $k=\left(k_{1}, \ldots, k_{n}\right), k_{j} \in \mathbb{Z}^{\ell_{j}}$, построено явное дивергентное представление $b^{\varepsilon}-b^{0}=$ $\sum \partial_{z_{i}} B_{i}$. Достаточное условие регулярности функции $\beta$ в терминах коэффициентов Фурье $\beta_{k}$ дается следующими двумя условиями:

$$
\begin{gathered}
\sum\left|\beta_{k}\right|_{L^{\infty}}\left|k_{j} \omega_{j}\right|^{-1}<\infty, \\
\sum\left|\partial_{x}^{1} \beta_{k}\right|_{L^{n}}\left|k_{j} \omega_{j}\right|^{-1}<\infty .
\end{gathered}
$$


Здесь суммирование происходит по всем целочисленным векторам $k=\left(k_{1}, \ldots, k_{n}\right) \in$ $\mathbb{Z}^{\ell}$ и $j=1, \ldots, n$, для которых $k_{j} \neq 0$. Ввиду диофантова условия $(3.12)$, эти сходимости можно гарантировать, если потребовать алгебраическое убьвание величин $\left|\beta_{k}\right|_{L^{\infty}},\left|\partial_{x}^{1} \beta_{k}\right|_{L^{n} \text { как }}$

$$
\left(1+\left|k_{j}\right|\right)^{-\left(\ell_{j}-1+\delta\right)}(1+|k|)^{-\ell+\delta^{\prime}}
$$

для некоторого $\delta^{\prime}>0$. Это условие обеспечивает (1.19), см. [7], [8].

В полной аналогии с (3.13), (3.14) условия на коэффищиенты Фурье $\Gamma_{k}(x)$, соответствующие $z$-квазипериодическому члену $g=g(x, z)$, записываются как

$$
\begin{array}{r}
\sum\left|\Gamma_{k}\right|_{L^{2}}\left|k_{j} \omega_{j}\right|^{-1}<\infty \\
\sum\left|\partial_{x}^{1} \Gamma_{k}\right|_{L^{2 n /(n+2)}}\left|k_{j} \omega_{j}\right|^{-1}<\infty .
\end{array}
$$

Условие алгебраического убывания (3.15) величин $\left|\Gamma_{k}\right|_{L^{2}}$ и $\left|\partial_{x}^{1} \Gamma_{k}\right|_{L^{2 n /(n+2)}}$ будет обеспечивать сходимость рядов.

Со сделанньми вьше замечаниями мы видим, как пространственно-квазипериодические коэффищиенты, частоты которых удовлетворяют диофантову условию, при некотором дополнительном условии дают пример гиперболических волновых уравнений с осциллируюшими коэффициентами, для которых справедливы наши теоремы.

\section{§4. Доказательство теорем 1.3 и 1.1}

В этом параграфе мы докажем теорему 1.3 о близости слабого решения $y^{\varepsilon}(t)$ уравнения $\frac{d}{d t} y^{\varepsilon}=A y^{\varepsilon}+\mathscr{F}_{\varepsilon}\left(y^{\varepsilon}\right)$, где $0 \leqslant \varepsilon \leqslant \varepsilon_{0}$, к решению $y^{0}(t)$ предельного (усредненного) уравнения (с $\varepsilon=0)$ с обшими начальными данными $y^{\varepsilon}(0)=y_{0} \in E_{0}$. В конще параграффа мы докажем теорему 1.1.

Напомним, что мы рассматриваем слабые решения в смысле интегро-операторного соотношения (1.38). Для доказательства повторно экспоненциальной оценки теоремы 1.3 мы рассмотрим разность

$$
w(t)=y^{\varepsilon}(t)-y^{0}(t)
$$

где $y^{0}(t) \in E_{0}$ удовлетворяет тому же интегральному соотношению (1.38), но с $\varepsilon=0$. Для разности $w(t)$ имеем $w(0)=0$ и, кроме того,

$$
w(t)=\int_{0}^{t} \exp (A(t-\tau))\left(\mathscr{F}_{\varepsilon}\left(y^{\varepsilon}(\tau)\right)-\mathscr{F}_{0}\left(y^{0}(\tau)\right)\right) d \tau
$$

в $E_{0}$. Чтобы получить повторно экспоненциальную оценку (1.43) порядка $\varepsilon^{\alpha}$ в промежуточном пространстве $E_{-\alpha}$, мы докажем неравенства

$$
\begin{aligned}
\|w(t)\|_{0} & \leqslant 2 M \exp \left(\varrho_{0} t\right) \\
\|w(t)\|_{-1} & \leqslant\left(\exp \left(\widetilde{c}_{2} \exp (\varrho t)+\widetilde{c}_{1} t\right)-1\right) \varepsilon
\end{aligned}
$$

с явными константами $\widetilde{c}_{1}, \widetilde{c}_{2}$, указанными ниже в $(4.15)$. Из этих неравенств с помошю интерполящии (1.34) мы получим неравенство для нормы пространства $E_{-\alpha}$, что 
докажет теорему 1.3, т.е. неравенство (1.43), с явно указанными в (1.44) постоянными $c_{0}, c_{1}, c_{2}$. Заметим, что неравенство (4.4) чуть-чуть сильнее, чем (1.43), где опущен внеэкспоненциальный член -1 , который становится несущественным при больших временах.

Неравенство (4.3) является прямым следствием отдельных экспоненциальных оценок $(1.39)$ для $y^{\varepsilon}(t)$ и $y^{0}(t)$ в $E_{0}$. Это неравенство дает лишь оценку, без какой-либо информации о сходимости при $\varepsilon \searrow 0$.

Для доказательства неравенства (4.4) в $E_{-1}$ мы применим лемму Гронуолла к соотношению (4.2). Для этого мы разобьем разность нелинейностей в подьштегральном выражении следующим образом:

$$
\mathscr{F}_{\varepsilon}\left(y^{\varepsilon}\right)-\mathscr{F}_{0}\left(y^{0}\right)=\left(\mathscr{F}_{\varepsilon}\left(y^{\varepsilon}\right)-\mathscr{F}_{0}\left(y^{\varepsilon}\right)\right)+\left(\mathscr{F}_{0}\left(y^{\varepsilon}\right)-\mathscr{F}_{0}\left(y^{0}\right)\right)
$$

Первая разность является величиной порядка $\varepsilon$ в $E_{-1}$. В самом деле, используя условие (1.42) и экспоненциальную оценку (1.39) для $y^{\varepsilon}(t)$, получаем

$$
\left\|\mathscr{F}_{\varepsilon}\left(y^{\varepsilon}(\tau)\right)-\mathscr{F}_{0}\left(y^{\varepsilon}(\tau)\right)\right\|_{-1} \leqslant C\left(1+M^{p_{0}} e^{\varrho_{0} p_{0} \tau}\right) \varepsilon .
$$

Аналогично, неравенство (1.41) дает

$$
\left\|\mathscr{F}_{0}\left(y^{\varepsilon}(\tau)\right)-\mathscr{F}_{0}\left(y^{0}(\tau)\right)\right\|_{-1} \leqslant C\left(1+M^{p_{1}} e^{\varrho_{0} p_{1} \tau}\right)\|w(\tau)\|_{-1} .
$$

Обозначим $p=\max \left(p_{0}, p_{1}\right), \varrho=\varrho_{0} p$ и

$$
c(\tau):=C\left(1+M^{p} e^{\varrho \tau}\right) .
$$

Тогда разбиение (4.5) и оценки (4.6), (4.7) влекут следующее неравенство:

$$
\left\|\mathscr{F}_{\varepsilon}\left(y^{\varepsilon}(\tau)\right)-\mathscr{F}_{0}\left(y^{0}(\tau)\right)\right\|_{-1} \leqslant c(\tau)\left(\|w(\tau)\|_{-1}+\varepsilon\right) .
$$

Подставляя это неравенство в соотношение (4.2) и используя (1.35), получаем следующее неравенство гронуолловского типа:

$$
\|w(t)\|_{-1} \leqslant \int_{0}^{t} M e^{\widetilde{\varrho}_{0}(t-\tau)} c(\tau)\left(\|w(\tau)\|_{-1}+\varepsilon\right) d \tau .
$$

Сведением к лемме Гронуолла мы сможем получить

$$
\|w(t)\|_{-1} \leqslant C(t) \varepsilon \text {. }
$$

Здесь мы использовали обозначение

$$
C(t)=\exp \left(\int_{0}^{t}\left(\widetilde{\varrho}_{0}+M c(\tau)\right) d \tau\right)-1,
$$

где $c(\tau)$ определено экспоненциальным выражением (4.8). Мы также без ограничения обшности предположили, что $\widetilde{\varrho}_{0} \geqslant 0$.

Для доказательства (4.11), (4.12) заметим, что функция

$$
\xi(t):=\left(1+\varepsilon^{-1}\|w(t)\|_{-1}\right) \exp \left(-\widetilde{\varrho}_{0} t\right)>0
$$


удовлетворяет $\xi(0)=1$ и

$$
\xi(t) \leqslant 1+\int_{0}^{t} M c(\tau) \xi(\tau) d \tau
$$

по (4.10). Теперь, используя лемму Гронуолла, мы получаем

$$
\xi(t) \leqslant \exp \left(\int_{0}^{t} M c(\tau) d \tau\right),
$$

что доказывает (4.11) с (4.12).

Прямое вычисление показьвает, что

$$
C(t) \leqslant \exp \left(\widetilde{c}_{2} e^{\varrho t}+\widetilde{c}_{1} t\right)-1
$$

с константами $\widetilde{c}_{1}, \widetilde{c}_{2}$, данными явными формулами:

$$
\begin{aligned}
& \widetilde{c}_{1}=M C+\widetilde{\varrho}_{0}, \\
& \widetilde{c}_{2}=\varrho^{-1} M^{p+1} C,
\end{aligned}
$$

где $\varrho>0$. Неравенства $(4.11),(4.14)$ доказьвают неравенство (4.4). Отметим консервативньй и диссипативньй случаи, для которых $\varrho_{0}=\varrho=0$ и функция $c(\tau)$ имеет вид $c(\tau)=C\left(1+M^{p}\right)$. В этом случае (4.4) упрошается до

$$
\|w(t)\|_{-1} \leqslant \exp \left(\widetilde{c}_{1}^{0} t\right) \varepsilon
$$

где

$$
\widetilde{c}_{1}^{0}=M C\left(1+M^{p}\right)+\widetilde{\varrho}_{0} .
$$

С помошюю интерполящии (1.34) неравенств (4.3) и (4.4), как было указано выше, получаем неравенство для нормы пространства $E_{-\alpha}$, что завершает доказательство теоремы 1.3, а также дополнения к этой теореме (1.46), (1.47).

Чтобы вьвести теорему 1.1 из теоремы 1.3, мы должны проверить справедливость предположений теоремы 1.3 в конкретном случае (1.48), (1.49), соответствующем гиперболическому волновому уравнению (1.1), (1.2). Мы должны проверить условия (1.34), (1.35), (1.39)-(1.42) теоремы 1.3. Интерполяционное неравенство (1.34) справедливо в силу нашего выбора пространств $E_{-\alpha}=H^{1-\alpha} \times H^{-\alpha}$. Оценка роста (1.39) доказана в $\S 2$. Экспоненциальная оценка (1.35) для полугруппы линейных операторов $\exp (A t)$ в $\mathscr{L}\left(E_{0}\right)$ также следует из результатов $\S 2$, если положить $f \equiv 0, g \equiv 0, \gamma \equiv 0$. Для пространства $\mathscr{L}\left(E_{-1}\right)$ справедливо то же самое неравенство. Оно получается из предыдущего сопряжением с помощью изоморфизма $(-\Delta)^{-1 / 2}: E_{0} \rightarrow E_{-1}$. Неравенства (1.42) и (1.41) были доказаны в $§ 3$. Поэтому теорему 1.3 действительно можно применить, что доказьвает повторно экспоненциальную оценку (1.22) теоремы 1.1 .

В случае $\gamma^{0} \geqslant \underline{\gamma}>0$ строго положительной диссипации мы утверждали справедливость более сильного неравенства (1.23) с одной экспонентой. Чтобы доказать это, рассмотрим вариант теоремы 1.3 с $\varrho=\varrho_{0}=0,(1.45)$, который дается неравенством (1.46) с (1.47). Априорная оценка $\left\|y^{\varepsilon}(t)\right\|_{0} \leqslant M\left(y_{0}\right)$, предположенная в (1.45), вьполняется при $\gamma^{0} \geqslant \underline{\gamma}>0$ и, более того, при $\gamma^{\varepsilon} \geqslant 0 ;$ см. предположения (1.21) и замечания в конще $\S 2$. (Аналогично получаем, что неравенство (1.35) для полугруппы $\exp (A t)$, на самом деле, вьполнено с нулевой скоростью роста $\widetilde{\varrho}_{0}=0$.) Из (1.46) получаем оценку роста (1.23) с одной экспонентой, что завершает доказательство теоремы 1.1. 


\section{$\S$ 5. Близость аттракторов к экспоненциальному}

В этом параграфе мы сначала докажем близость глобальных аттракторов допредельных уравнений в промежуточных пространствах к глобальному аттрактору предельного уравнения в случае, когда последний является экспоненциально притягивающим, а именно, мы докажем неравенство (1.32), которое утверждается теоремой 1.2. Доказательство (см. лемму 5.1) основано на экспоненциальном притяжении решений при $\varepsilon=0$ к глобальному аттрактору $\mathscr{A}^{0}$, которое отмечено в (1.31). Такой абстрактный подход был известен и ранее, см., например, [10], [7]. Затем мы применим результаты, полученные Бабиным и Вишиком [3], чтобы доказать требуемое экспоненциальное притяжение аттрактора $\mathscr{A}^{0}$ в случае гиперболического волнового уравнения (1.1) с краевыми условиями (1.2) в пространстве $E_{0}=H_{0}^{1} \times L^{2}$. См. также [6].

Мы изложим доказательство неравенства (1.32) в абстрактной форме, следуя изложению в [7; лемма 4.1]. Пусть для каждого $0 \leqslant \varepsilon \leqslant \varepsilon_{0}$ задана полугруппа $y^{\varepsilon}(t)$, $t \geqslant 0$, действуюшая в банаховом пространстве $E_{-\alpha}$, причем для любого начального положения $y_{0}$ неравенство

$$
\left\|y^{\varepsilon}(t)-y^{0}(t)\right\|_{-\alpha} \leqslant C \varepsilon^{\alpha} e^{\varrho t}
$$

вьполнено равномерно для всех $0 \leqslant \varepsilon \leqslant \varepsilon_{0}, t \geqslant 0$. Константы $C$ и $\varrho$ могут зависеть от $\alpha$ и от нормы $\left\|y_{0}\right\|_{0}$ начального положения $y_{0}=y^{\varepsilon}(0)=y^{0}(0)$, но не от $\varepsilon$. Мы сейчас не требуем выполнения интерполяционных неравенств для пространств $E_{-\alpha}$. Но мы предполагаем, что пространства $E_{-\alpha}$ являются расширением основного пространства $E_{0} \subseteq E_{-\alpha}$ и, соответственно, что норма пространства $E_{0}$, которой мы вьше измеряли начальные данные, является более сильной, чем нормы в пространствах $E_{-\alpha}$.

Пусть $\mathscr{A}^{\varepsilon}, 0 \leqslant \varepsilon \leqslant \varepsilon^{0}$, обозначает семейство обратно инвариантных подпространств в $E_{0} \subseteq E_{-\alpha}$ в следуюшем смысле: для каждого $\widetilde{y}_{0} \in \mathscr{A}^{\varepsilon}$ и любого $t \geqslant 0$ существует некоторое начальное состояние $y_{0} \in \mathscr{A}^{\varepsilon}$ такое, что для решения $y^{\varepsilon}$ в $E_{0}$ с начальным состоянием $y^{\varepsilon}(0)=y_{0}$ вьполнено $y^{\varepsilon}(t)=\widetilde{y}_{0}$. Мы также предполагаем экспоненциальную скорость притяжения к $\mathscr{A}^{0}$ в $E_{-\alpha}$ :

$$
\operatorname{dist}_{E_{-\alpha}}\left(y^{0}(t), \mathscr{A}^{0}\right) \leqslant C e^{-\nu t}
$$

равномерно по всем начальным положениям

$$
y_{0} \in \bigcup_{0 \leqslant \varepsilon \leqslant \varepsilon_{0}} \mathscr{A}^{\varepsilon} \subseteq E_{0} \subseteq E_{-\alpha} .
$$

Наконец, мы предположим, что в пространстве $E_{-\alpha}$ условие (5.1) вьполнено равномерно по всем начальным состояниям $y_{0}$ из объединения аттракторов (5.3). В силу того, что ранее мы уже предположили условие (5.1), достаточно предположить ограниченность множества $\bigcup \mathscr{A}^{\varepsilon}$ в основном пространстве $E_{0}$ с более сильной нормой $\|\cdot\|_{0}$.

ЛЕмма 5.1. При сделанных выше предположсениях (5.1) и (5.2) существует $\varepsilon$-независимая константа $C>0$ такая, что неравенство (1.32) выполнено $c$ показателем (1.33). Величины $\alpha, \varrho, \nu$ введены выше в (5.1), (5.2). 
ДокАЗАТЕльство (см. [7]). Доказательство состоит в простом применении неравенства треугольника. Пусть $0<\varepsilon \leqslant \varepsilon_{0}<1$. Для каждого пространства $\mathscr{A}^{\varepsilon} \subseteq E_{0}$ положим $t:=-\nu^{-1} \alpha^{\prime} \log \varepsilon>0$. Согласно обратной инвариантности для каждого $\widetilde{y}_{0} \in \mathscr{A}^{\varepsilon}$ выберем $y_{0} \in \mathscr{A}^{\varepsilon}$ такое, что $y^{\varepsilon}(t)=\widetilde{y}_{0}$. Тогда неравенства $(5.1),(5.2)$ и выбор $t$ влекут

$$
\begin{aligned}
\operatorname{dist}_{E_{-\alpha}}\left(\widetilde{y}_{0}, \mathscr{A}^{0}\right) & =\operatorname{dist}_{E_{-\alpha}}\left(y^{\varepsilon}(t), \mathscr{A}^{0}\right) \\
& \leqslant\left\|y^{\varepsilon}(t)-y^{0}(t)\right\|_{-\alpha}+\operatorname{dist}_{E_{-\alpha}}\left(y^{0}(t), \mathscr{A}^{0}\right) \\
& \leqslant C\left(\varepsilon^{\alpha} e^{\varrho t}+e^{-\nu t}\right)=2 C \varepsilon^{\alpha^{\prime}} .
\end{aligned}
$$

Лемма 5.1 доказана.

Пусть $\mathscr{A}^{\varepsilon}$ - глобальный аттрактор в пространстве $E_{0}=H_{0}^{1} \times L^{2}$ полугруппы $S^{\varepsilon}(t)$ решений $y^{\varepsilon}=\left(u^{\varepsilon}, u_{t}^{\varepsilon}\right) \in E_{0}$, соответствуюшей гиперболическому волновому уравнению (1.1), (1.2). Аналогично, пусть $\mathscr{A}^{0}-$ глобальный аттрактор разрешаюшей полугрупшы $S^{0}(t)$ усредненного уравнения (1.14).

Для доказательства теоремы 1.2 нам осталось проверить справедливость предположений леммы 5.1 в случае $(1.48),(1.49)$, соответствующем гиперболическому волновому уравнению (1.1), (1.2). Условие (5.1) доказано в теореме 1.1, (1.23); см. конец $\S$. Экспоненциальная скорость притяжения (5.2) будет установлена ниже, даже для более сильной нормы пространства $E_{0}$.

Опишем необходимые для этого доводы. Более ранние и технически более ограничительные подходы можно найти в [17], [3], [6], [15], [5]. Используя топологию пространства $E_{0}=H_{0}^{1} \times L^{2}$, отметим и частично прокомментируем следующие факты:

(i) непрерывность зависимости $y^{0}\left(t ; y_{0}\right)$ от $\left(t, y_{0}\right) \in[0, \infty) \times E_{0}$;

(ii) существование глобальных аттракторов $\mathscr{A}^{\varepsilon}$ и $\mathscr{A}^{0}$;

(iii) предкомпактность множества $\bigcup_{0 \leqslant \varepsilon \leqslant \varepsilon_{0}} \mathscr{A}^{\varepsilon}$ в $E_{0}$;

(iv) липшицевость в $E_{0}$

$$
\left\|y^{1}(t)-y^{2}(t)\right\|_{0} \leqslant C e^{\widetilde{\varrho} t}\left\|y_{0}^{1}-y_{0}^{2}\right\|_{0}
$$

зависимости решений $y^{1}, y^{2}$ при $\varepsilon=0$ от начальных данных $y_{0}^{1}, y_{0}^{2}$ в окрестности $\mathscr{V}\left(\mathscr{A}^{0}\right)$ аттрактора $\mathscr{A}^{0}\left(C\right.$ и $\widetilde{\varrho}$ зависят только от $\left.\mathscr{V}\left(\mathscr{A}^{0}\right)\right) ;$

(v) непрерывность убьвания гамильтониана (энергии) $\Phi$ в $E_{0}$, см. (1.27);

(vi) конечность и гиперболичность стационарных решений при $\varepsilon=0$;

(vii) $C^{1+\theta}$-зависимость по норме $E_{0}$ решений $y^{0}\left(t ; y_{0}\right)$ при $\varepsilon=0$ от начальных данных $y_{0}$ при $t=0$ в окрестности указанных стационарных точек.

В [3] показано, что в совокупности свойства (i), (ii), (iv)-(vii) влекут экспоненциальную сходимость относительно компактных множеств $K$ к глобальному аттрактору $\mathscr{A}^{0}$ при $\varepsilon=0$ по норме $E_{0}$. Но согласно свойству (iii), доказанному ниже, $K:=$ $\bigcup \mathscr{A}^{\varepsilon}$ является предкомпактным множеством. Это докажет свойство экспоненциального притяжения (5.2). Поэтому чтобы доказать теорему 1.2 , достаточно убедиться в справедливости утверждений (i)-(vii), сформулированных вьше.

Начнем с непрерьвной зависимости решения $S^{\varepsilon}(t) y_{0}=y^{\varepsilon}\left(t ; y_{0}\right)$ от $\left(t, y_{0}\right) \in$ $[0, \infty) \times E_{0}$, которая утверждается в (i). Это обший факт теории полулинейных уравнений (сформулированньй здесь в обозначениях (1.48), (1.49), соответствующих нашему гиперболическому волновому уравнению), которьй следует из локальной 
липшицевости абстрактной нелинейности $\mathscr{F}_{\varepsilon}(y)$ в $E_{0}$; см. $(2.3)$ и, например, [16], [14], [3; теорема 7.1].

Теперь покажем существование (ii) и предкомпактность (iii) глобальных аттракторов. Полугруппы $S^{\varepsilon}(t)$ в $E_{0}$ обладают $\varepsilon$-независимым поглощающим множеством $\mathscr{B}$, которое компактно в $E_{0}$. Таким множеством является шар достаточно большого радиуса $R$ в $E_{\sigma}$, где $\sigma:=1-\frac{1}{2}(p-1)(n-2)>0$. При $n=3$ см. [19; с. 204-206]. При $n \geqslant 3$ см. также [9], [11]. Доказательство в [19] переносится дословно на случай быстро осциллирующих коэффициентов $b(x, x / \varepsilon), g(x, x / \varepsilon)$, потому что в нем ни разу не производится дифференцирование по $x$. Поэтому глобальные аттракторы $\mathscr{A}^{\varepsilon}$ сушествуют при $0 \leqslant \varepsilon \leqslant \varepsilon_{0}$. Их объединение предкомпактно в $E_{0}$, поскольку оно содержится в $\varepsilon$-независимом множестве $\mathscr{B}$, которое компактно в $E_{0}$. Это доказьвает свойства (ii), (iii).

Свойство (iv) следует из равномерной ограниченности в $E_{0}$ решений с начальными данньми в $\mathscr{V}\left(\mathscr{A}^{0}\right)$. Доказательство использует оценку роста $(1.8)$ производной $f^{\prime}(u)$ и проводится методами $\S 2$.

Свойство непрерьвности (v) гамильтониана $\Phi$ следует из оценки роста (1.8), проинтегрированной дважды по $u$, и соболевского вложения $H_{0}^{1} \hookrightarrow L^{2 n /(n-2)}$.

Свойство (vi) уже предположено в теореме 1.2 .

Условие Гёльдера $(1.26)$ на $f^{\prime}(u)$ влечет соответствующее свойство гёльдеровости для абстрактной нелинейности $\mathscr{F}(u)$ по норме $E_{0}$ и, следовательно, обеспечивает свойство гёльдеровости соответствующей полугруппы, которое утверждается в (vii).

Это завершает доказательство свойств (i)-(vii).

Подытожим наше доказательство теоремы 1.2 об оценках сходимости глобальных аттракторов $\mathscr{A}^{\varepsilon}$ диссипативного гиперболического волнового уравнения (1.1) с краевыми условиями (1.2). Согласно [3] свойства (i)-(vii) влекут равномерную по $\varepsilon$ экспоненциальную скорость сходимости

$$
\operatorname{dist}_{H_{0}^{1} \times L^{2}}\left(S^{0}(t)\left(\bigcup_{0 \leqslant \varepsilon \leqslant \varepsilon} \mathscr{A}^{\varepsilon}\right), \mathscr{A}^{0}\right) \leqslant C e^{-\nu t}
$$

для предельной полугрупшы $S^{0}(t)$, с подходящими константами $C, \nu>0$. Это доказывает, что вьполнено условие (5.2) леммы 5.1. Напомним, что условие (5.1) леммы 5.1 следует из теоремы 1.1 (см. (1.23)). В терминах $\left(u, u_{t}\right)=y \in E_{-\alpha}=H^{1-\alpha} \times H^{-\alpha}$ оно означает

$$
\left\|u^{\varepsilon}(t, \cdot)-u^{0}(t, \cdot)\right\|_{H^{1-\alpha}}+\left\|u_{t}^{\varepsilon}(t, \cdot)-u_{t}^{0}(t, \cdot)\right\|_{H^{-\alpha}} \leqslant C \varepsilon^{\alpha} e^{\varrho t}
$$

для всех $0 \leqslant \alpha \leqslant 1$ и подходяших констант $C, \varrho>0$. Оба условия леммы требуют положительности диссипативного коэффициента. Из леммы 5.1 теперь следует неравенство

$$
\operatorname{dist}_{H^{1-\alpha} \times H^{-\alpha}}\left(\mathscr{A}^{\varepsilon}, \mathscr{A}^{0}\right) \leqslant C e^{\alpha^{\prime}}
$$

для подходящей константы $C$ и $\alpha^{\prime}:=\alpha /(1+\varrho / \nu)$. Теорема 1.2 доказана, поскольку (5.8) эквивалентно (1.32).

В качестве заключения мы упомянем открытую проблему оценок сходимости аттракторов в случае предельного показателя

$$
p=n /(n-2) \text {, }
$$


которьй включает случай $p=3$ кубической нелинейности $f$, когда размерность пространства равна $n=3$. Известно, что в предельном случае глобальньй аттрактор $\mathscr{A}^{0}$, а также отдельно каждый из аттракторов $\mathscr{A}^{\varepsilon}$ остаются компактными в $E_{0}$, cм. [3], [1] и [15]. Но, поскольку свойство (iii) предкомпактности множества

$$
\bigcup_{0 \leqslant \varepsilon \leqslant \varepsilon_{0}} \mathscr{A}^{\varepsilon}
$$

в $E_{0}$ не известно, представленное нами доказательство не покрьвает случай предельного показателя $p$.

\section{СПИСОК ЛИТЕРАТУРЫ}

[1] J. Arrieta, A. N. Carvalho, J. K. Hale. A damped hyperbolic equation with critical exponent // Comm. Partial Differential Equations. 1992. V. 17. № 5/6. P. 841-866.

[2] A. V. Babin, M. I. Vishik. Regular attractors of semigroups and evolution equations // J. Math. Pures Appl. (9). 1983. V. 62. № 4. P. 441-491.

[3] А. В. Бабин, М. И. Вишик. Аттракторы эволюционных уравнений. М.: Наука, 1989.

[4] J. W.S. Cassels. An Introduction to Diophantine Approximation. Cambridge: Cambridge Univ. Press, 1957. (Cambridge Tracts in Math. Math. Phys. № 45.)

[5] V. V. Chepyzhov, M.I. Vishik. Attractors for Equations of Mathematical Physics. Providence, RI: Amer. Math. Soc., 2002. (Amer. Math. Soc. Colloq. Publ. V. 49.)

[6] A. Eden, C. Foias, B. Nicolaenko, R. Temam. Exponential Attractors for Dissipative Evolution Equations. Chichester/Paris: Wiley/Masson, 1994. (Res. Appl. Math. V. 37.)

[7] B. Fiedler, M. Vishik. Quantitative homogenization of global attractors for reaction-diffusion systems with rapidly oscillating terms // Preprint, 2000.

[8] B. Fiedler, M. Vishik. Quantitative homogenization of analytic semigroups and reaction-diffusion equations with Diophantine spatial frequencies // Adv. Differential Equations. 2001. V. 6. № 11. P. 1377-1408.

[9] J. Hale. Asymptotic behaviour and dynamics in infinite dimensions // Res. Notes Math. 1985. V. 132. P. 1-42.

[10] J. K. Hale, G. Raugel. Lower semicontinuity of attractors of gradient systems and applications // Ann. Mat. Pura Appl. 1989. V. 154. P. 281-326.

[11] A. Haraux. Two remarks on hyperbolic dissipative problems // Res. Notes Math. 1985. V. 122. P. 161-179.

[12] D. Henry. Geometric Theory of Semilinear Parabolic Equations. New York: Springer-Verlag, 1981. (Lecture Notes in Math. V. 840.)

[13] T. Kato. Perturbation Theory for Linear Operators. Berlin: Springer-Verlag, 1966. (Grundlehren Math. Wiss. V. 132.)

[14] A. Pazy. Semigroups of Linear Operators and Applications to Partial Differential Equations. New York: Springer-Verlag, 1983. (Appl. Math. Sci. V. 44.)

[15] G. Raugel. Global attractors of partial differential equations // Handbook of Dynamical Systems / ed. B. Fiedler. V. 2. Amsterdam: Elsevier, 2002.

[16] H. Tanabe. Equations of Evolution. London: Pitman, 1979.

[17] R. Temam. Infinite-Dimensional Dynamical Systems in Mechanics and Physics. New York: Springer-Verlag, 1988. (Appl. Math. Sci. V. 68.) 